\title{
Serum level and polymorphisms of retinol- binding protein-4 and risk for gestational diabetes mellitus: a meta-analysis
}

\author{
Shimin $\mathrm{Hu}^{1}$, Qian Liu ${ }^{1,2}$, Xin Huang ${ }^{1}$ and Hongzhuan $\operatorname{Tan}^{1 *}$
}

\begin{abstract}
Background: Retinol-binding protein-4 (RBP4) has been reported to be potentially involved in the pathogenesis of gestational diabetes mellitus (GDM); however, the findings are inconsistent. Our aims were to review the studies that investigated the association of serum levels and polymorphisms of RBP4 with GDM risk, and to provide recommendations for future research.

Methods: The databases PubMed, EBSCO, ScienceDirect, and Web of Knowledge were searched up to October 2015 to find out studies evaluating the relationship between serum RBP4 level/ RBP4 polymorphisms and GDM risk. In the meta-analysis of serum RBP4 levels the key inclusion was that studies were designed as BMI-matched studies or had observed non-significant differences in BMl between cases and controls.

Results: Fourteen case-control studies (647 cases and 620 controls) reporting the association between serum RBP4 level and GDM risk, and three studies (1012 cases and 1605 controls) investigating the association between RBP4 polymorphisms and GDM risk were involved. Our results showed that high serum RBP4 levels represent a risk factor for GDM (pooled standardized mean difference $=0.758,95 \%$ confidence interval [0.387, 1.128]). The results of subgroup analyses based on "gestational age at blood sampling" or "diagnostic criteria" are consistent with the overall results. However, the postpartum subgroup and "before 24 weeks" subgroup both only include one article and indicate no association between serum RBP4 level and GDM risk. The meta-analysis on the association between rs3758539 polymorphism and GDM risk shows that RBP4 rs3758539 polymorphism is not associated with the development of GDM.
\end{abstract}

Conclusions: The results of this meta-analysis support the hypothesis that RBP4 is a modest independent risk factor for GDM (i.e., nonobese patients with GDM might express RBP4 at abnormal levels). The serum RBP4 level is associated with the risk of GDM. However, the association in the first-trimester and postpartum period should be validated by further research. The association between RBP4 rs3758539 polymorphism and GDM risk was not confirmed.

Keywords: Retinol-binding protein-4, Gestational diabetes mellitus, Meta-analysis

\section{Background}

Gestational diabetes mellitus (GDM) is defined as varying degrees of glucose intolerance that is first detected during pregnancy [1]. The prevalence of GDM is increasing in decades and floating from 1.7 to $11.6 \%$ among populations [2]. Although considerable research effort has been focused on GDM, the pathophysiology of

\footnotetext{
*Correspondence: tanhz99@qq.com

'Department of Epidemiology and Health Statistics, School of Public Health

of Central South University, Changsha, Hunan 410007, PR China

Full list of author information is available at the end of the article
}

the disease remains incompletely understood. During pregnancy, insulin resistance will be enhanced physiologically. Insulin resistance can be further strengthened by some factors such as obesity, leading to high risk of GDM [3-5].

Retinol-Binding Protein-4 (RBP4) is mainly synthesized by hepatocytes and adipose tissues. It was identified in 2005 as an adipocytokine with potential capability in reducing insulin sensitivity and enhancing hepatic gluconeogenesis [6]. A multi-center clinical study [7] revealed that serum RBP4 levels correlated with the magnitude of 
insulin resistance in subjects with obesity, impaired glucose tolerance, or type 2 diabetes and in nonobese, nondiabetic subjects with a strong family history of type 2 diabetes. In nonobese subjects, decreased expression of glucose transporter type 4 (GLUT-4) in adipocytes predicts increased serum RBP4 levels and insulin resistance. Adipose-specific deletion of GLUT-4 was reported to lead to secondary defects in insulin action in muscle and liver. The mechanism by which a decrease in adipocyte GLUT4 results in an increase in RBP4 expression is unknown, but it might involve sensing of glucose by adipocytes. RBP4 is regulated by GLUT-4. The downstream of RBP4's regulatory mechanism is that an increase in serum RBP4 levels can induce hepatic expression of phosphoenolpyruvate carboxykinase, a gluconeogenic enzyme, to increase gluconeogenesis, and impair insulin signaling in muscles through decreasing the expression of phosphoinositide- 3 kinase [8].

In patients with a history of GDM, insulin resistance exists before pregnancy but worsens during gestation. Insulin secretion is insufficient to compensate for the insulin resistance and this leads to hyperglycemia, which can be detected through routine glucose screening during pregnancy. Thus, chronic insulin resistance is a central component of the pathophysiology of GDM [9]. Furthermore, the cellular GLUT-4 content also decreases in patients with GDM [10]. The aforementioned findings have predictably given rise to the hypothesis that RBP4 might contribute to insulin resistance in GDM, much as it does in type 2 diabetes.

To date, several genetic variants that affect RBP4 expression levels (e.g., rs3758539 and rs12265684) have been investigated for their potential association with the risk of GDM, but the reported findings are inconsistent [11-13]. False-negative results, inadequate statistical power, small sample sizes, and ethnic differences may contribute to the lack of reproducibility in geneticassociation studies [14, 15]. Among the RBP4 genetic variants examined in the studies to date, only the rs3758539 variant was analyzed more than once, in the USA, China, and Mexico, and therefore we only analyzed the association between RBP4 genetic variants (rs3758539) and GDM in the present study.

The association between serum RBP4 levels and GDM risk has also been investigated, and, the reported results are also inconsistent [12, 13, 16-33]. Statistical association does not necessarily mean causal association. We propose 3 hypotheses regarding the positive statistical association between RBP4 levels and GDM risk: (1) GDM pathophysiology is related to obesity but not RBP4 levels, and serum RBP4 levels are elevated because of the increased size of adipocytes [7]; (2) RBP4 is a pathophysiological link between obesity and insulin resistance; (3) RBP4 is a modest independent risk factor for GDM (i.e., nonobese patients with GDM might express RBP4 at abnormal levels). If we want to prove serum RBP4 level is associated with GDM risk, considerable attention must be paid to body mass index (BMI) matched strategy. The positive results of studies with BMI-matched strategy are more credible to convince us that RBP4 is a modest independent risk factor for GDM. In meta-analysis about serum RBP4 level, we only included those studies in which BMI was matched, or showed a similar distribution in case and control groups, and then analyzed whether RBP4 is an independent risk factor for GDM.

Our aims were to review previous studies that investigated the association of serum RBP4 levels and RBP4 polymorphisms with GDM risk, and to provide recommendations for future research.

\section{Methods}

\section{Literature search}

First, the databases PubMed, EBSCO, ScienceDirect, and Web of Knowledge were searched up to October 2015 to find out studies evaluating the relationship between serum RBP4 level and GDM risk. The following keywords were used: ("gestational diabetes mellitus" OR "gestational diabetes" OR GDM) AND ("retinol-binding protein-4" OR "retinol binding protein-4" OR "retinol binding protein 4" OR "retinol-binding protein 4" OR RBP4 OR RBP-4). Next, we systematically searched the same databases for studies that examined RBP4 polymorphisms in patients with GDM. The following keywords were used in this step: ("gestational diabetes mellitus" OR "gestational diabetes" OR "GDM") AND ("polymorphism" OR "variant") AND ("retinol-binding protein-4" OR "retinol binding protein-4" OR "retinol binding protein 4" OR "retinol-binding protein 4" OR "RBP4" OR "RBP-4"); furthermore, the names of specific polymorphisms were combined with "gestational diabetes." All reference lists of the resulting primary research reports and relevant reviews were manually searched to identify additional eligible studies.

\section{Eligible studies and data extraction}

Eligible studies included in this meta-analysis: (1) investigated the relationship between serum RBP4 levels and GDM risk, or between GDM and at least 1 genetic variant of RBP4; (2) included a case group of patients with GDM and a control group; (3) diagnosed GDM according to the oral glucose-tolerance test (OGTT); (4) provided data with mean and standard deviation (SD) or median and interquartile range (in the case of the meta-analysis of serum RBP4 levels); (5) were not animal studies; (6) were designed as BMI-matched studies or had observed nonsignificant differences in BMI between cases and controls (in the case of the meta-analysis of serum RBP4 levels). 
Studies with overlapping data were excluded from our analysis. We identified only one study that required additional information: Ping et al. [12] had not reported the $P$ value of the difference in BMI between cases and controls in their analysis of serum RBP4 levels. However, we obtained Ping's $\mathrm{PhD}$ dissertation, written in Chinese, and extracted this information from it. Data were extracted independently by two reviewers in consultation with a third, from each study included. The information regarding the first author, year of publication, study population (country and ethnicity), number of patients and controls, and diagnostic criteria were extracted. For genetic-association studies, information was extracted on the frequency of genotypes, the HardyWeinberg equilibrium (HWE) status, and the genotyping method used. For studies investigated serum RBP4 levels, information was extracted on serum RBP4 measurements, the mean and SD of serum RBP4 levels, and the gestational age at the time of blood sampling. When the standard error of the mean (SEM) was reported, SD was calculated as $S D=S E M / \sqrt{n}$. If the case or control groups were further divided into subgroups, the data from the subgroups were merged as $\mathrm{n}=\mathrm{n}_{1}+\mathrm{n}_{2}, \bar{x}=\frac{n_{1} \overline{1}+n_{2} \overline{x_{2}}}{n_{1}+n_{2}}$

and $\quad \mathrm{SD}=\sqrt{\frac{\left(n_{1}-1\right) S D_{1}^{2}+\left(n_{2}-1\right) S D_{2}^{2}+\frac{n_{1} n_{2}}{n_{1}+n_{2}}\left(\bar{x}_{1}^{2}+\bar{x}_{2}^{2}-2 \bar{x}_{1} \bar{x}_{2}\right)}{n_{1}+n_{2}-1}}$

When a study provided medians and interquartile ranges (instead of means and SDs), without the minimum or maximum values, we treated the medians as means and calculated the SDs, as $\mathrm{SD}=$ interquartile range/1.35 [35]. If the study provided the minimum and maximum, we imputed the means and SDs as described by Hozo et al. [36]. When a study had tested the blood sample by two or more different methods, the result of the enzyme immunometric assay was chosen here because it is the most commonly used method in the studies included in our meta-analysis.

\section{Statistical methods}

The association between serum RBP4 level and GDM risk was estimated by calculating the pooled standardized mean difference (SMD) and $95 \%$ confidence interval (CI). To analyze the potential influences of gestational age at blood sampling and diagnostic criteria, we performed subgroup analysis. Based on the gestational age at blood sampling, the subjects were divided into 4 subgroups: (1) before 24 weeks, (2) 24-28 weeks, (3) after 28 weeks, and (4) postpartum; For two studies $[26,28]$, the gestational ages at blood sampling considerably overlapped within 24-28 weeks; thus, these two studies were assigned to the gestational age subgroup of 24-28 weeks. And according to the diagnostic criteria studies were divided into two subgroups: (1) based on the American Diabetes Association (ADA) criteria [37], and (2) based on the more rigorous criteria established by the National Diabetes Data Group (NDDG) [38], the World Health Organization (WHO) [39], and Sun et al. [20]. The association between genetic factors (e.g., genotypes and alleles) and GDM was examined by using the Chi-square test or the Fisher exact test. The association of the RBP4 rs3758539 polymorphism with GDM risk was assessed, by calculating the pooled odds ratio (OR) and $95 \% \mathrm{CI}$, according to general, dominant, and recessive genetic models, and an allelic model $[40,41]$. The significance of the pooled OR and SMD was determined using $\mathrm{Z}$ test, and the level was set at $p<0.05$.

Heterogeneity among studies was assessed using the $Q$ test and the $I^{2}$ statistic $[42,43]$. When significant heterogeneity was observed $\left(P<0.1\right.$ in the $Q$ test, and $I^{2}>$ $50 \%$ ), a random effects model was used for pooling data from the primary studies; if the heterogeneity was not significant, a fixed effects model was used. The HWE compliance of the controls in each study was assessed using the Chi-square test. Sensitivity analysis was performed by sequentially excluding individual studies to assess the stability of the results. Funnel-plot asymmetry was assessed using Egger's linear regression test, $P<0.05$ representing significant publication bias. If asymmetry was observed, contour-enhanced meta-analysis funnel plots were used to distinguish publication bias from other causes of asymmetry $[44,45]$. All analyses were performed using STATA 12.0 software (Stata Corporation, College Station, TX), and all $p$ values were 2-tailed.

\section{Results}

\section{Main characteristics of eligible studies}

With respect to the association between serum RBP4 levels and GDM risk, an initial search identified 232 records of potentially relevant studies from the databases included. Of these, 202 records were excluded based on their title and/or abstract: these were repetitive publications, reviews, reports of animal studies, and/or reports of studies that investigated either outcomes irrelevant to this meta-analysis or adipokines other than RBP4. A further 16 full-text articles were excluded because: (1) six papers contain data that overlapped with other articles, (2) seven papers reported different BMI distributions between the case and control groups $(P<0.05)$, (3) and three papers with the plasma RBP4 concentrations or unavailable data. Final selected 14 case-control studies included 647 cases and 620 controls were included in our meta-analysis (Table 1, Additional file 1: Table S1); the process of study selection is shown in Additional file 2: Figure $\mathrm{S} 1(\mathrm{a})$.

With respect to the association between $R B P 4$ rs3758539 polymorphism and GDM risk, the initial database search identified 147 reports of potentially relevant studies. Of these, 143 records were excluded based on their title and/ 
Table 1 Detailed characteristics of all eligible studies for the association with serum RBP4 levels and GDM

\begin{tabular}{|c|c|c|c|c|c|c|c|c|c|c|c|}
\hline \multirow[t]{2}{*}{ Study } & \multirow[t]{2}{*}{ Year } & \multirow[t]{2}{*}{ Country } & \multirow{2}{*}{$\begin{array}{l}\text { No of } \\
\text { case }\end{array}$} & \multirow{2}{*}{$\begin{array}{l}\text { No of } \\
\text { control }\end{array}$} & \multirow{2}{*}{$\begin{array}{l}\text { Diagnose } \\
\text { criteriab }^{\text {b }}\end{array}$} & \multirow{2}{*}{$\begin{array}{l}\text { Gestational age at } \\
\text { blood sampling }\end{array}$} & \multirow[t]{2}{*}{ Test } & \multirow[t]{2}{*}{$\mathrm{BMl}^{\mathrm{c}}$} & \multicolumn{2}{|l|}{$\mathrm{RBP} 4(\mu \mathrm{g} / \mathrm{ml})^{\mathrm{d}}$} & \multirow[t]{2}{*}{$P$-value } \\
\hline & & & & & & & & & Case & Control & \\
\hline Chan a [16] & 2007 & China, Han & 20 & 20 & NDDG & 24-28 weeks & ELISA & Matched & $0.0424 \pm 0.0138$ & $0.0320 \pm 0.0087$ & 0.007 \\
\hline Chan b [16] & 2007 & China, Han & 20 & 20 & NDDG & $\begin{array}{l}\text { immediately after } \\
\text { delivery }\end{array}$ & ELISA & Matched & $0.0301 \pm 0.0110$ & $0.0309 \pm 0.0100$ & 0.811 \\
\hline Kim [17] & 2008 & South Korea & 10 & 9 & ADA & 24-28 weeks & ELISA & $P>0.05$ & $39.1 \pm 6.3$ & $30.0 \pm 10.0$ & 0.026 \\
\hline $\begin{array}{l}\text { Lewandowski }^{\text {a }} \\
{[19]}\end{array}$ & 2008 & Austria & 15 & 35 & ADA & 28 weeks & Unclear & Matched & $53.9 \pm 18.7$ & $34.4 \pm 12.4$ & $<0.001$ \\
\hline Sun [20] & 2009 & China, Han & 32 & 30 & $\begin{array}{l}\text { OGTT } \\
\text { confirmed }\end{array}$ & $\begin{array}{l}24 \mathrm{~h} \text { before } \\
\text { delivery }\end{array}$ & ELISA & Matched & $27.0 \pm 1.2$ & $19.4 \pm 1.8$ & $<0.001$ \\
\hline Klein a [21] & 2010 & Austria, Caucasian & 63 & 38 & ADA & 24-28 weeks & ELISA & Matched & $18.0 \pm 3.7$ & $16.9 \pm 5.1$ & 0.213 \\
\hline Klein b [21] & 2010 & Austria, Caucasian & 63 & 38 & ADA & 33 weeks & ELISA & Matched & $20.3 \pm 7.2$ & $17.9 \pm 6.1$ & 0.089 \\
\hline Su [23] & 2010 & China, $\operatorname{Han}$ & 63 & 58 & ADA & 24-28 weeks & ELISA & Matched & $41.6 \pm 12.2$ & $34.5 \pm 9.8$ & $<0.001$ \\
\hline Tepper [24] & 2010 & USA, mixed & 12 & 10 & ADA & 24-28 weeks & EIA & Matched & $25.2 \pm 2.1$ & $25.2 \pm 2.1$ & 1.000 \\
\hline $\begin{array}{l}\text { Kuzmicki a } \\
{[26]}\end{array}$ & 2011 & Poland & 68 & 68 & $\mathrm{WHO}$ & 24-30 weeks & EIA & $P>0.05$ & $58.1 \pm 25.3$ & $51.0 \pm 18.1$ & 0.062 \\
\hline $\begin{array}{l}\text { Kuzmicki ba } \\
{[26]}\end{array}$ & 2011 & Poland & 20 & 18 & $\mathrm{WHO}$ & 36-40 weeks & EIA & $P>0.05$ & $62.4 \pm 31.0$ & $42.9 \pm 19.0$ & 0.027 \\
\hline Chen [25] & 2011 & China, Han & 52 & 46 & NDDG & before delivery & ELISA & Matched & $31.93 \pm 7.21$ & $26.92 \pm 8.5$ & 0.002 \\
\hline Ping [12] & 2012 & China, $\operatorname{Han}$ & 74 & 69 & ADA & 24-28 weeks & ELISA & $P>0.05$ & $21.53 \pm 5.96$ & $20.84 \pm 4.31$ & $<0.001$ \\
\hline Skvarca ${ }^{a}[28]$ & 2012 & Slovenia & 30 & 44 & ADA & $\begin{array}{l}26.81 \pm \\
3.46 \text { weeks }\end{array}$ & ELISA & $P>0.05$ & $15.00 \pm 6.11$ & $15.57 \pm 8.35$ & 0.750 \\
\hline Liang [31] & 2014 & China, Han & 35 & 35 & NDDG & 24-28 weeks & ELISA & $P>0.05$ & $22.90 \pm 3.09$ & $17.90 \pm 3.91$ & $<0.001$ \\
\hline Fruscalzo [32] & 2015 & Germany, mixed & 32 & 44 & ADA & 11-13 weeks & ELISA & $P>0.05$ & $24.78 \pm 6.51$ & $27.93 \pm 7.98$ & 0.071 \\
\hline Du [33] & 2015 & China & 38 & 38 & NDDG & 37-42 weeks & ELISA & Matched & $39.08 \pm 8.29$ & $21.42 \pm 3.85$ & $<0.001$ \\
\hline
\end{tabular}

${ }^{a}$ Raw data in the article are presented as median (interquartile range)

${ }^{b}$ Diagnostic criteria: NDDG National Diabetes Data Group criteria, $A D A$, American Diabetes Association criteria, WHO World Health Organization criteria 'BMI: matched, the control subjects had been matched with the GDM subjects for BMl; $P>0.05$, difference of $B M I$ between case and control had no statistical significance

${ }^{\mathrm{d} D a t a}$ are presented as mean \pm standard deviation

Table 2 Detailed characteristics of all eligible studies for the association with RBP4 gene polymorphisms and GDM

\begin{tabular}{|c|c|c|c|c|c|c|c|c|c|c|}
\hline \multirow{2}{*}{$\begin{array}{l}\text { Gene } \\
\text { polymorphisms }\end{array}$} & \multirow[t]{2}{*}{ Author } & \multirow[t]{2}{*}{ Year } & \multirow[t]{2}{*}{ Country } & \multirow[t]{2}{*}{ Ethnicity } & \multirow[t]{2}{*}{ Case include } & \multirow{2}{*}{$\begin{array}{l}\text { No of } \\
\text { case }\end{array}$} & \multirow{2}{*}{$\begin{array}{l}\text { No of } \\
\text { control }\end{array}$} & \multicolumn{2}{|l|}{ Genotype $^{a}$} & \multirow{2}{*}{$\begin{array}{l}P \text { for } \\
\text { HWE }\end{array}$} \\
\hline & & & & & & & & Case & Control & \\
\hline \multirow[t]{5}{*}{ rs3758539 } & Hiraoka $a^{d}[11]$ & 2011 & America & Utah Caucasian & GDM & 88 & 315 & $56 / 31 / 1$ & 228/77/10 & 0.27 \\
\hline & Hiraoka b ${ }^{d}[11]$ & 2011 & America & Hawai'i Filipino & GDM & 82 & 286 & $63 / 16 / 3$ & $226 / 55 / 5$ & 0.44 \\
\hline & Hiraoka $c^{d}[11]$ & 2011 & America & Hawai'i Pacific Islander & GDM & 19 & 32 & 18/1/0 & 23/9/0 & 0.35 \\
\hline & Ping $^{\mathrm{e}}[12]$ & 2012 & China & Han & $\mathrm{GDM}+\mathrm{GIGT}^{\mathrm{c}}$ & 723 & 872 & $375 / 75 / 5$ & $462 / 143 / 8$ & 0.41 \\
\hline & Saucedo $^{d}[13]$ & 2014 & Mexico & Unclear & GDM & 100 & 100 & $93 / 7$ & $91 / 9$ & $P>0.05^{b}$ \\
\hline rs12265684 & Ping $^{e}$ [12] & 2012 & China & Han & $\mathrm{GDM}+\mathrm{GIGT}^{\mathrm{c}}$ & 723 & 872 & $382 / 106 / 5$ & $483 / 168 / 14$ & 0.89 \\
\hline rs3758538 & Ping $^{d}[12]$ & 2012 & China & Han & $\mathrm{GDM}+\mathrm{GIGT}^{\mathrm{c}}$ & 723 & 872 & $414 / 63 / 4$ & $562 / 76 / 2$ & 0.74 \\
\hline rs10882273 & Ping $^{d}[12]$ & 2012 & China & Han & $\mathrm{GDM}+\mathrm{GIGT}^{\mathrm{c}}$ & 723 & 872 & $363 / 107 / 8$ & $451 / 174 / 13$ & 0.42 \\
\hline rs116736522 & Saucedo ${ }^{d}[13]$ & 2014 & Mexico & Unclear & GDM & 100 & 100 & $97 / 3$ & $96 / 4$ & $P>0.05^{b}$ \\
\hline rs34571439 & Saucedo ${ }^{d}[13]$ & 2014 & Mexico & Unclear & GDM & 100 & 100 & $91 / 9$ & $88 / 12$ & $P>0.05^{b}$ \\
\hline
\end{tabular}

a Genotype for rs3758539, GG/AG/AA (For Saucedo, GG/GA + AA); rs12265684, CC/CG/GG; rs3758538, AA/AC/CC; rs10882273, TT/TC/CC; rs116736522, GG/GC + CC; rs34571439, AA/AC + CC

${ }^{\mathrm{b}}$ No sufficient data to calculate the P for HWE. Authors mentioned "all variants are in the Hardy-Weinberg equilibrium" in the article

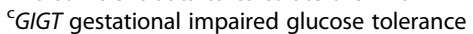

${ }^{\mathrm{d}}$ No significant differences was found in allelic frequencies between case and control groups

esignificant differences was found in allelic frequencies between case and control groups 
Table 3 Summary of different comparative results of serum RBP4 level with GDM risk

\begin{tabular}{|c|c|c|c|c|c|c|c|c|c|}
\hline Category & & No of study & No of case & No of control & $\mathrm{SMD}(95 \% \mathrm{Cl})$ & Z & $p$ value & $1^{2} \%$ & $P_{\text {het }}$ \\
\hline overall & & 17 & 647 & 620 & $0.816[0.411-1.122]$ & 3.94 & 0.000 & 91.0 & 0.000 \\
\hline \multirow{4}{*}{$\begin{array}{l}\text { Gestational age at blood } \\
\text { sampling }\end{array}$} & Before 24 weeks & 1 & 32 & 44 & $-0.426[-0.886-0.035]$ & 1.81 & 0.070 & - & - \\
\hline & 24-28 weeks & 10 & 390 & 386 & $0.561[0.252-0.870]$ & 3.56 & 0.000 & 74.8 & 0.000 \\
\hline & After 28 weeks & 5 & 205 & 170 & $1.830[0.580-3.080]$ & 2.87 & 0.004 & 96.0 & 0.000 \\
\hline & Postpartum & 1 & 20 & 20 & $-0.076[-0.696-0.544]$ & 0.24 & 0.810 & - & - \\
\hline \multirow[t]{2}{*}{ Diagnostic criteria } & ADA criteria & 8 & 347 & 310 & $0.205[-0.063-0.472]$ & 1.50 & 0.134 & 61.7 & 0.011 \\
\hline & Rigorous criteria & 9 & 300 & 310 & 1.388 [0.665-2.110] & 3.77 & 0.000 & 91.0 & 0.000 \\
\hline
\end{tabular}

$P_{\text {het }}=p$ value for heterogeneity, $O R=$ adds ratio, $C l=$ confidence interval, $A D A=$ American Diabetes Association criteria, More rigorous criteria contained National Diabetes Data Group criteria, World Health Organization criteria and the criteria of Sun et al

or abstract: these were repetitive publications, reviews, reports of animal studies, and/or reports of studies that investigated either outcomes irrelevant to this meta-analysis or genes other than RBP4. In addition, 1 full-text review was excluded because it contained data overlapped with other primary papers. Finally, 3 eligible studies that included 1,012 cases and 1,605 controls were included in the meta-analysis (Table 2); the process of study selection is shown in Additional file 2: Figure S1(b).

\section{Association between serum RBP4 level and GDM Risk}

The meta-analysis for serum RBP4 level includes 14 articles (17 results) with a total of 647 cases and 620 controls. The results show that serum RBP4 level and GDM risk are significantly associated (SMD $=0.816,95 \% \mathrm{CI}$ [0.411, 1.122]) (Table 3, Fig. 1). The "24-28 weeks" subgroup, "after 28 weeks" subgroup and the rigorous criteria subgroup indicate that higher serum RBP4 level is related to GDM risk (24-28 weeks subgroup:

\begin{tabular}{|c|c|c|c|c|c|}
\hline Study & & & $\mathrm{N}$, mean & $\mathrm{N}$, mean & $\%$ \\
\hline ID & & $\operatorname{SMD}(95 \% \mathrm{Cl})$ & (SD); Treatment & (SD); Control & Weight \\
\hline Chan a (2007) & $\rightarrow-$ & $0.90(0.25,1.55)$ & $20, .0424(.0138)$ & $20, .032(.0087)$ & 5.73 \\
\hline Chan b (2007) & -1 & $-0.08(-0.70,0.54)$ & $20, .0301(.011)$ & $20, .0309(.01)$ & 5.81 \\
\hline $\operatorname{Kim}(2008)$ & — & $1.10(0.13,2.08)$ & $10,39.1(6.3)$ & $9,30(10)$ & 4.84 \\
\hline Lewandowski (2008) & & $1.34(0.68,2.00)$ & $15,53.9(18.7)$ & $35,34.4(12.4)$ & 5.70 \\
\hline Sun (2009) & & $5.00(3.98,6.02)$ & $32,27(1.2)$ & $30,19.4(1.8)$ & 4.70 \\
\hline Klein a (2010) & & $0.26(-0.15,0.66)$ & $63,18(3.7)$ & $38,16.9(5.1)$ & 6.30 \\
\hline Klein b (2010) & & $0.35(-0.05,0.76)$ & $63,20.3(7.2)$ & $38,17.9(6.1)$ & 6.30 \\
\hline Su (2010) & $\rightarrow$ & $0.64(0.27,1.00)$ & $63,41.6(12.2)$ & $58,34.5(9.8)$ & 6.37 \\
\hline Tepper (2010) & -1 & $0.00(-0.84,0.84)$ & $12,25.2(2.1)$ & $10,25.2(2.1)$ & 5.22 \\
\hline Kuzmicki a (2011) & - & $0.32(-0.02,0.66)$ & $68,58.1(25.3)$ & $68,51(18.1)$ & 6.42 \\
\hline Kuzmicki b (2011) & & $0.75(0.09,1.41)$ & $20,62.4(31)$ & $18,42.9(19)$ & 5.70 \\
\hline chen (2011) & $\rightarrow$ & $0.64(0.23,1.05)$ & $52,31.9(7.21)$ & $46,26.9(8.5)$ & 6.29 \\
\hline Ping (2012) & & $0.13(-0.20,0.46)$ & $74,21.5(5.96)$ & $69,20.8(4.31)$ & 6.44 \\
\hline Skvarca (2012) & 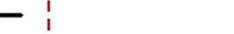 & $-0.08(-0.54,0.39)$ & $30,15(6.11)$ & $44,15.6(8.35)$ & 6.18 \\
\hline Liang (2014) & $1 \rightarrow$ & $1.42(0.89,1.94)$ & $35,22.9(3.09)$ & $35,17.9(3.91)$ & 6.04 \\
\hline Fruscalzo (2015) & & $-0.43(-0.89,0.03)$ & $32,24.8(6.51)$ & $44,27.9(7.98)$ & 6.18 \\
\hline Du (2015) & $\longrightarrow$ & $2.73(2.10,3.36)$ & $38,39.1(8.29)$ & $38,21.4(3.85)$ & 5.78 \\
\hline Overall (1-squared $=91.0 \%, p=0.000$ ) & & $0.82(0.41,1.22)$ & 647 & 620 & 100.00 \\
\hline NOTE: Weights are from random effects analysis & & & & & \\
\hline $\begin{array}{c}1 \\
-6.02\end{array}$ & & & & & \\
\hline
\end{tabular}


$\mathrm{SMD}=0.561,95 \%$ CI [0.252, 0.870]; after 28 weeks: $\mathrm{SMD}=1.830,95 \% \mathrm{CI}[0.580-3.080]$; the rigorous criteria subgroup: $\mathrm{SMD}=1.388,95 \% \mathrm{CI}[0.665,2.110]$ ). The "before 24 weeks" subgroup and postpartum subgroup both include only 1 study and show non-significant association between serum RBP4 level and GDM risk (before 24 weeks subgroup: $\mathrm{SMD}=-0.426,95 \% \mathrm{CI}$ $[-0.886,0.870]$; postpartum subgroup SMD $=-0.076,95 \%$ CI $[-0.696,0.472])$. Non-significant result is also observed in the ADA criteria subgroup $(\mathrm{SMD}=0.205,95 \% \mathrm{CI}$ [-0.063, 0.472]) (Table 3, Figs. 2 and 3).

In the sensitivity analysis, 1 eligible study was excluded at a time in order to assess the influence of each dataset on the pooled SMD. We observed no changes in the corresponding pooled SMD or in the significance of the results (Additional file 3: Figure S2), which indicated that our results were significantly robust to the studyselection process.

Publication bias was assessed using Begg's funnel plot and Egger's test. The results of Begg's funnel plot reveal the presence of significant asymmetry. These results are consistent with the modified Egger linear regression test and Begg's test $(t=2.50, p=0.025 ; Z=2.27, p=0.023)$. In order to analyze the potential causes to the funnel plot asymmetry, we treated the contour-enhanced funnel plots with the trim-and-fill method. This treatment resulted in 4 filled studies, which were all in the region of $p<0.05$ (Fig. 4). Hence, the cause of the asymmetry may be more likely to be due to factors other than publication bias, such as variable study quality. After the "unpublished" (filled) studies are included, higher serum RBP4 level is still related to GDM risk (SMD $=0.300,95 \%$ CI $[0.189,0.411]) . "$

\section{Association between rs3758539 Polymorphisms and GDM Risk}

The meta-analysis concerning rs3758539 included three studies with a total of 1012 cases and 1605 controls. The dominant genetic model shows non-significant association between GG vs. GA + AA (OR $=1.133,95 \%$ CI $[0.695,1.846])$. The following results were obtained only including two studies [8, 9]: $\mathrm{G}$ allele vs. A allele

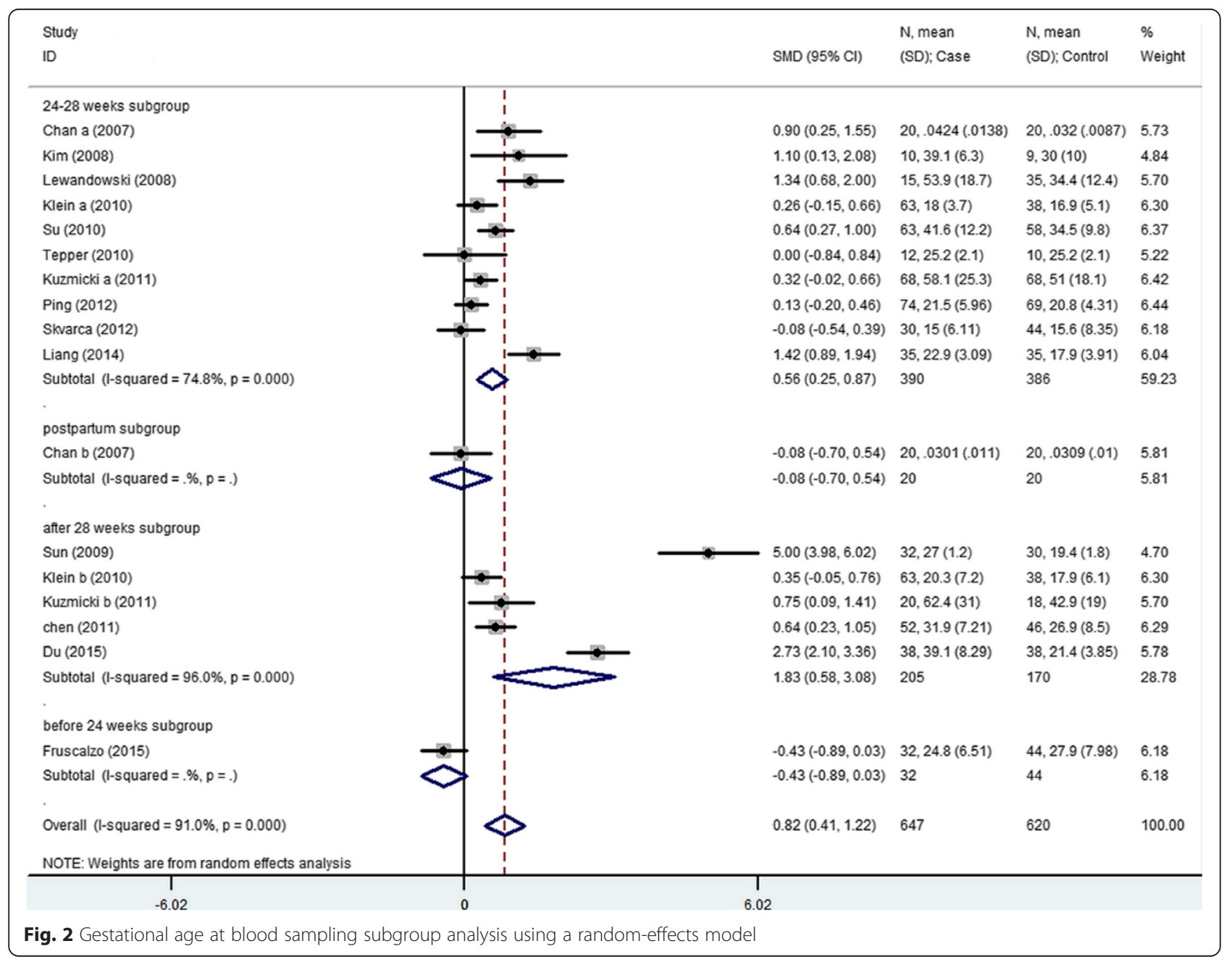




\begin{tabular}{|c|c|c|c|c|c|}
\hline \multicolumn{2}{|l|}{ Study } & & $\mathrm{N}$, mean & $\mathrm{N}$, mean & $\%$ \\
\hline \multicolumn{2}{|l|}{ ID } & $\operatorname{SMD}(95 \% \mathrm{Cl})$ & (SD); Case & (SD); Control & Weight \\
\hline \multicolumn{6}{|l|}{ rigorous criteria subgroup } \\
\hline Chan a (2007) & & $0.90(0.25,1.55)$ & $20, .0424(.0138)$ & $20, .032(.0087)$ & 5.73 \\
\hline Chan b (2007) & - & $-0.08(-0.70,0.54)$ & $20, .0301(.011)$ & $20, .0309(.01)$ & 5.81 \\
\hline Lewandowski (2008) & & $1.34(0.68,2.00)$ & $15,53.9(18.7)$ & $35,34.4(12.4)$ & 5.70 \\
\hline Sun (2009) & 1 & $5.00(3.98,6.02)$ & $32,27(1.2)$ & $30,19.4(1.8)$ & 4.70 \\
\hline Kuzmicki a (2011) & & $0.32(-0.02,0.66)$ & $68,58.1(25.3)$ & $68,51(18.1)$ & 6.42 \\
\hline Kurmicki b (2011) & $\rightarrow$ & $0.75(0.09,1.41)$ & $20,62.4(31)$ & $18,42.9(19)$ & 5.70 \\
\hline chen (2011) & & $0.64(0.23,1.05)$ & $52,31.9(7.21)$ & $46,26.9(8.5)$ & 6.29 \\
\hline Liang (2014) & $1 \rightarrow$ & $1.42(0.89,1.94)$ & $35,22.9(3.09)$ & $35,17.9(3.91)$ & 6.04 \\
\hline Du (2015) & $\longrightarrow$ & $2.73(2.10,3.36)$ & $38,39.1(8.29)$ & $38,21.4(3.85)$ & 5.78 \\
\hline Subtotal (1-squared $=93.4 \%, p=0.000$ ) & & $1.39(0.67,2.11)$ & 300 & 310 & 52.18 \\
\hline \multicolumn{6}{|l|}{. } \\
\hline \multicolumn{6}{|l|}{ ADA criteria subgroup } \\
\hline $\operatorname{Kim}(2008)$ & & $1.10(0.13,2.08)$ & $10,39.1(6.3)$ & $9,30(10)$ & 4.84 \\
\hline Klein a (2010) & $-i$ & $0.26(-0.15,0.66)$ & $63,18(3.7)$ & $38,16.9(5.1)$ & 6.30 \\
\hline Klein b (2010) & 7 & $0.35(-0.05,0.76)$ & $63,20.3(7.2)$ & $38,17.9(6.1)$ & 6.30 \\
\hline Su (2010) & $\rightarrow$ & $0.64(0.27,1.00)$ & $63,41.6(12.2)$ & $58,34.5(9.8)$ & 6.37 \\
\hline Tepper (2010) & & $0.00(-0.84,0.84)$ & $12,25.2(2.1)$ & $10,25.2(2.1)$ & 5.22 \\
\hline Ping (2012) & & $0.13(-0.20,0.46)$ & $74,21.5(5.96)$ & $69,20.8(4.31)$ & 6.44 \\
\hline Skvarca (2012) & - & $-0.08(-0.54,0.39)$ & $30,15(6.11)$ & $44,15.6(8.35)$ & 6.18 \\
\hline Fruscalzo (2015) & 1 & $-0.43(-0.89,0.03)$ & $32,24.8(6.51)$ & $44,27.9(7.98)$ & 6.18 \\
\hline Subtotal $(1-$ squared $=61.7 \%, p=0.011)$ & 1 & $0.20(-0.06,0.47)$ & 347 & 310 & 47.82 \\
\hline \multicolumn{6}{|l|}{. } \\
\hline Overall (I-squared $=91.0 \%, p=0.000$ ) & & $0.82(0.41,1.22)$ & 647 & 620 & 100.00 \\
\hline NOTE: Weights are from random effects analysis & & & & & \\
\hline 1 & & & & & \\
\hline
\end{tabular}

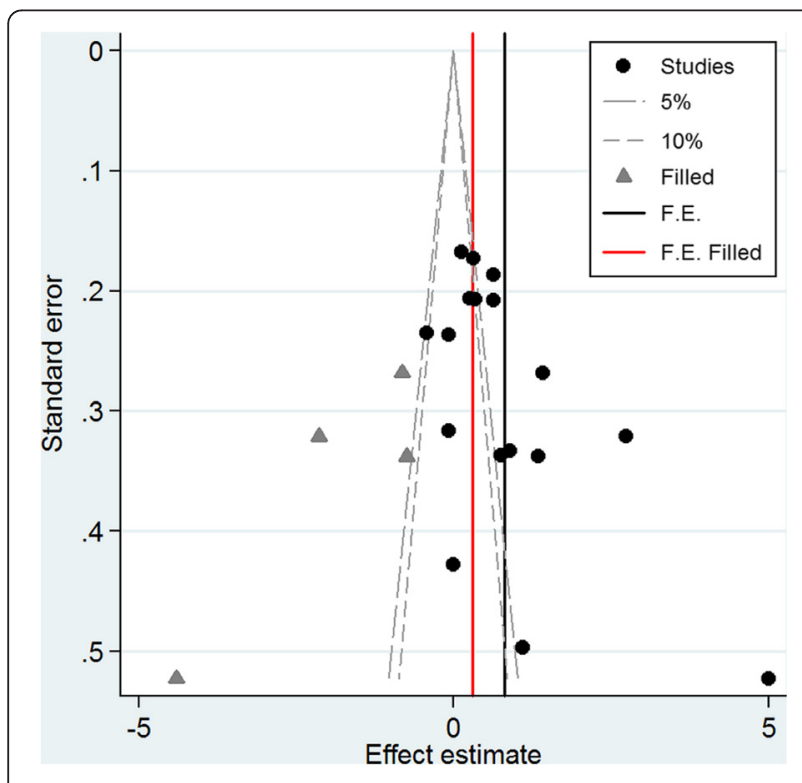

Fig. 4 Contour-enhanced funnel plots for funnel plot asymmetry analysis of serum RBP4 level with GDM risk
$(\mathrm{OR}=1.093,95 \%$ CI $[0.687,1.739]), \mathrm{GG}+\mathrm{GA}$ vs. AA $(\mathrm{OR}=1.123,95 \% \mathrm{CI}[0.508,2.483])$, and $\mathrm{GG}$ vs. AA $(\mathrm{OR}=1.124,95 \%$ CI $[0.505,2.502])$ (Table 4, Fig. 5). Publication bias was not detected owing to the small number of available studies. During the sensitivity analysis, one eligible study was excluded each time to investigate the influence of the individual dataset on the pooled OR. The results show that the corresponding pooled OR and significant results did not change materially (Additional file 4: Figure S3), indicating that our results were significantly robust.

\section{Discussion}

Main findings

Our meta-analysis shows that higher prenatal serum RBP4 level is related to GDM risk, but this association cannot be shown in the first-trimester and postpartum periods. The GDM diagnostic criteria affected the strength of association between RBP4 level and GDM risk. Adopting a higher threshold of OGTT would result in a larger difference of serum RBP4 level between GDM 
Table 4 Summary of different comparative results of rs3758539 polymorphism

\begin{tabular}{|c|c|c|c|c|c|c|c|c|c|}
\hline \multicolumn{2}{|c|}{ Genetic model } & \multirow{2}{*}{$\frac{\text { No of case }}{1012}$} & \multirow{2}{*}{$\frac{\text { No of control }}{1605}$} & \multirow{2}{*}{$\frac{\mathrm{OR}(95 \% \mathrm{Cl})}{1.133[0.695-1.846]}$} & \multirow{2}{*}{$\frac{Z}{0.50}$} & \multirow{2}{*}{$\frac{p \text { value }}{0.617}$} & \multirow{2}{*}{$\frac{1^{2} \%}{65.2}$} & \multirow{2}{*}{$\frac{P_{\text {het }}}{0.022}$} & \multirow{2}{*}{$\frac{\text { Effect model }}{R}$} \\
\hline Dominant & $\mathrm{GG}$ versus $\mathrm{GA}+\mathrm{AA}$ & & & & & & & & \\
\hline Recessive & $\mathrm{GG}+\mathrm{GA}$ versus $\mathrm{AA}$ & 893 & 1473 & $1.123[0.508-2.483]$ & 0.29 & 0.775 & 8.3 & 0.336 & $\mathrm{~F}$ \\
\hline Additive & GG versus $A A$ & 893 & 1473 & $1.124[0.505-2.502]$ & 0.29 & 0.775 & 0.9 & 0.365 & $\mathrm{~F}$ \\
\hline Allele & $G$ versus $A$ & 912 & 1505 & $1.093[0.687-1.739]$ & 0.37 & 0.709 & 68.3 & 0.024 & $\mathrm{R}$ \\
\hline
\end{tabular}

$P_{\text {het }}=p$ value for heterogeneity, $O R=$ adds ratio, $C l=$ confidence interval, $F=$ fixed-effect model, $R=$ random-effect model

women and controls. RBP4 rs3758539 polymorphisms may not be associated with GDM risk.

\section{Interpretation}

We pooled 17 studies (647 cases and 620 controls) in which the BMI-matched strategy was used, and then analyzed the relationship between serum RBP4 levels and GDM risk. Our results indicated that high serum RBP4 levels were related to GDM risk. We performed subgroup analyses of the effects of gestational age at blood sampling and diagnostic criteria. The studies included in the meta-analysis covered all periods during the pregnancy and only one study concerned the postpartum situation. The pooled results of second- and thirdtrimester subgroups were consistent with the overall result, GDM gravidas have higher serum RBP4 level than the controls. No statistically significant association was found between serum RBP4 levels and GDM risk in the postpartum subgroup and first-trimester subgroup. In the postpartum study blood samples were collected immediately after delivery. High exertion at delivery, which requires high output of energy, might temporarily enhance insulin sensitivity [16], and specific metabolic state at delivery might produce a difference between postpartum and prenatal results. The negative result of first-trimester subgroup reveals that with the insulin resistance level increasing during pregnancy, the magnitude of the effect of RBP4 may change. However, the inclusion of only a single study in postpartum subgroup and "before 24 weeks" subgroup could potentially make the stratified analysis unreliable. Therefore, the results must be interpreted with caution, and need to be validated using additional studies that include a large number of samples covering various periods during pregnancy and after delivery. In the subgroup analyses based on diagnostic criteria, both of the two subgroups show results consistent with the overall meta-analysis. And the pooled SMD of rigorous criteria subgroup is

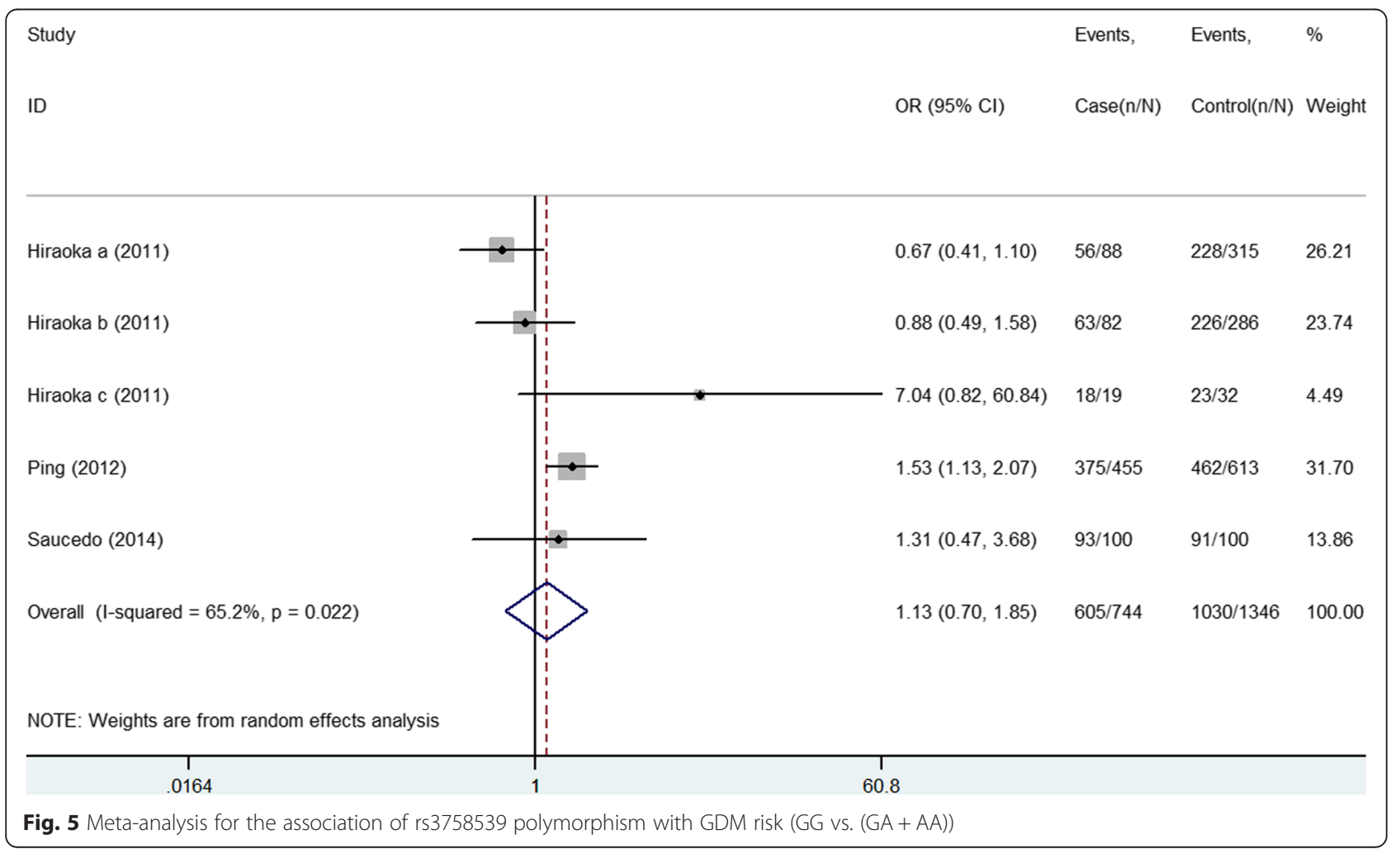


higher than the pooled SMD of ADA criteria subgroup. Although the result of ADA criteria subgroup had no statistical significance, we still can get further hints: higher blood glucose level at fasting and OGTT is associated with higher serum RBP4 levels. This finding was consistent with several published articles [17, 18, 21, 23, 31], which revealed that blood glucose and serum RBP4 levels presents a modest dose-response relationship. Thus, the results of subgroup analysis based on diagnostic criteria confirmed our hypothesis that RBP4 is related to glycometabolism in GDM.

Two articles reported the odds ratio about serum RBP4 level between women with and without GDM estimated from logistic regression models. The study by Abetew et al. [29] revealed that women in the highest quartile for serum RBP4 had a 1.89-fold higher risk of GDM compared with women in the lowest quartile $(95 \%$ CI: $1.05-$ 3.43). However, this relationship did not reach statistical significance after adjustment for maternal race/ethnicity, family history of diabetes, and pre-pregnancy overweight status (adjusted OR: 1.54; 95 \% CI: 0.82-2.90). In the study by Fruscalzo et al. [32], serum RBP4 level was not involved in the final multivariate logistic regression model after adjustment for maternal age, pre-pregnancy BMI, tobacco smoke, macro-region of origin, familial history of diabetes and so on.

The coding region of RBP4 is located at chromosome 10q23-24 in humans, contains 5 exons and 6 introns, and has been linked to increased risk for type 2 diabetes in various populations [46, 47]. Numerous single nucleotide polymorphisms (SNPs), including +5,398 $\mathrm{C}>\mathrm{T},+8,201 \mathrm{~T}>\mathrm{A},+8,204 \mathrm{~T}>\mathrm{A}$, rs17484721, rs36035572, rs3758539, rs3758539, rs10882273, rs36014035, and rs34571439, have been identified as genetic markers for type 2 diabetes or insulin resistance [48-56]. Some of these SNPs are associated with circulating RBP4 level or RBP4 expression in visceral adipose tissues [48, 55, 57]. In this study, we investigated the relationship between the RBP4 rs3758539 polymorphism and susceptibility to GDM, but did not find any significant association. Among the five races included in the meta-analysis, significant results were only observed in Han Chinese. However, given the low frequency of the minor allele of rs3758539 in these ethnic groups [11, 13] and the small OR [12], non-significant results could be attributed to the small sample size. Therefore, additional studies of a comparatively larger scale are required to further validate this result.

To the best of our knowledge, this is the first metaanalysis that has evaluated the relationship between genetic variants of RBP4 and the risk of GDM. However, the study has certain limitations. First, the subgroup analysis performed based on gestational age at blood sampling included only one postpartum study, which makes the stratified analysis unreliable. Moreover, the meta-analysis of RBP4 polymorphisms involved a small number of samples and studies. Therefore, the results must be interpreted with caution. Second, funnel-plot asymmetry can confound the interpretation of metaanalyses. Here, the observed funnel-plot asymmetry might have resulted from poor methodological quality related to the small size and heterogeneity of the studies, in addition to publication bias $[35,44,45]$. Third, the sample size of each individual study of RBP4 levels and GDM is relatively small and all these studies are case-control studies. These factors affect the quality of individual studies included in the meta-analysis. Last, our study only included articles that featured English-language abstracts, and the main text written in English or Chinese, and this might have resulted in a language bias.

\section{Conclusions}

The results of this meta-analysis support the hypothesis that RBP4 is a modest independent risk factor for GDM (i.e., nonobese patients with GDM might express RBP4 at abnormal levels). The serum RBP4 level is associated with the risk of GDM. However, the association in the first-trimester and postpartum period should be validated by further research. The association between RBP4 rs3758539 polymorphism and GDM risk was not confirmed.

\section{Additional files}

Additional file 1: Table S1. Adjusted covariates of all eligible studies for the association with serum RBP4 levels and GDM. (DOCX 18 kb)

Additional file 2: Figure S1. PRISMA flow diagram of study selection process (a) genetic variants and (b) serum concentration. (TIFF 4988 kb)

Additional file 3: Figure S2. The results of sensitivity analysis of serum RBP4 level with GDM risk. (TIFF 8567 kb)

Additional file 4: Figure S3. The results of sensitivity analysis of rs3758539 (GG vs. GA + AA) with GDM risk. (TIFF 5448 kb)

\section{Abbreviations}

ADA: American Diabetes Association; BMl: body mass index; Cl: confidence interval; GDM: gestational diabetes mellitus; HWE: Hardy-Weinberg equilibrium; NDDG: National Diabetes Data Group; OGTT: oral glucose tolerance test; OR: odds ratio; PEPCK: phosphoenolpyruvate carboxykinase; RBP4: retinol-binding protein-4; SD: standard deviation; SEM: standard error of mean; SMD: standardized mean difference; SNPs: single nucleotide polymorphisms; WHO: World Health Organization.

\section{Competing interests}

The authors declare that they have no competing interests.

\section{Authors' contributions}

$\mathrm{SH}$ and QL has searched the databases and extracted the information. $\mathrm{XH}$, as the third reviewer, reviewed the processes of literature search and data extraction. SH did statistical analysis. SH and HT drafted the manuscript. All authors read and approved the final manuscript. 


\section{Author details}

${ }^{1}$ Department of Epidemiology and Health Statistics, School of Public Health of Central South University, Changsha, Hunan 410007, PR China. ${ }^{2}$ Criminal Investigation Division, Changsha Public Security Bureau, Hunan 410004, China.

Received: 31 January 2015 Accepted: 3 March 2016

Published online: 14 March 2016

\section{References}

1. Buchanan TA, Xiang AH. Gestational diabetes mellitus. J Clin Invest. 2005; 115(3):485-91.

2. Schneider S, Bock C, Wetzel M, Maul H, Loerbroks A. The prevalence of gestational diabetes in advanced economies. J Perinat Med. 2012;40(5):511-20.

3. Stupin $\mathrm{JH}$, Arabin B. Overweight and obesity before, during and after pregnancy: Part 1: pathophysiology, molecular biology and epigenetic consequences. Geburtshilfe Frauenheilkd. 2014;74(7):639-45.

4. Athukorala C, Rumbold AR, Willson KJ, Crowther CA. The risk of adverse pregnancy outcomes in women who are overweight or obese. BMC Pregnancy Childbirth. 2010;10:56

5. Ovesen P, Rasmussen S, Kesmodel U. Effect of prepregnancy maternal overweight and obesity on pregnancy outcome. Obstet Gynecol. 2011; 118(2 Pt 1):305-12.

6. Yang Q, Graham TE, Mody N, Preitner F, Peroni OD, Zabolotny JM, Kotani K, Quadro L, Kahn BB. Serum retinol binding protein 4 contributes to insulin resistance in obesity and type 2 diabetes. Nature. 2005:436(7049):356-62

7. Graham TE, Yang Q, Bluher M, Hammarstedt A, Ciaraldi TP, Henry RR, Wason CJ, Oberbach A, Jansson PA, Smith U, et al. Retinol-binding protein 4 and insulin resistance in lean, obese, and diabetic subjects. N Engl J Med. 2006; 354(24):2552-63.

8. Tamori Y, Sakaue H, Kasuga M. RBP4, an unexpected adipokine. Nat Med. 2006;12(1):30-1.

9. Barbour LA, McCurdy CE, Hernandez TL, Kirwan JP, Catalano PM, Friedman JE. Cellular mechanisms for insulin resistance in normal pregnancy and gestational diabetes. Diabetes Care. 2007;30 Suppl 2:S112-9.

10. Garvey WT, Maianu L, Zhu JH, Hancock JA, Golichowski AM. Multiple defects in the adipocyte glucose transport system cause cellular insulin resistance in gestational diabetes. Heterogeneity in the number and a novel abnormality in subcellular localization of GLUT4 glucose transporters. Diabetes. 1993; 42(12):1773-85.

11. Hiraoka M, Urschitz J, Sultan O, Ward K. A polymorphism in the retino binding protein 4 gene is not associated with gestational diabetes mellitus in several different ethnic groups. Hawaii Med J. 2011;70(8):164-7.

12. Ping F, Xiang HD, Li M, Li W, Liu JT, Nie M, Hui YC. Effects of variation in retinol binding protein 4 gene and adipose specific expression of gestational diabetes in Beijing, China. Diabetes Res Clin Pract. 2012; 97(2):283-9

13. Saucedo R, Zarate A, Basurto L, Hernandez M, Puello E, Mendoza-Lorenzo P, Ostrosky-Wegman P. RBP4 gene variants are associated with insulin resistance in women with previous gestational diabetes. Dis Markers. 2014; 2014:269208.

14. Hirschhorn JN, Lohmueller K, Byrne E, Hirschhorn K. A comprehensive review of genetic association studies. Genet Med. 2002;4(2):45-61.

15. Cardon LR, Bell Jl. Association study designs for complex diseases. Nat Rev Genet. 2001;2(2):91-9.

16. Chan TF, Chen HS, Chen YC, Lee CH, Chou FH, Chen IJ, Chen SY, Jong SB, Tsai EM. Increased serum retinol-binding protein 4 concentrations in women with gestational diabetes mellitus. Reprod Sci. 2007;14(2):169-74.

17. Kim SH, Choi HJ, Im JA. Retinol-binding protein 4 responses during an oral glucose tolerance testing in women with gestational diabetes mellitus. Clin Chim Acta. 2008;391(1-2):123-5.

18. Krzyzanowska K, Zemany L, Krugluger W, Schernthaner GH, Mittermayer F, Schnack C, Rahman R, Brix J, Kahn BB, Schernthaner G. Serum concentrations of retinol-binding protein 4 in women with and without gestational diabetes. Diabetologia. 2008;51(7):1115-22.

19. Lewandowski KC, Stojanovic N, Bienkiewicz M, Tan BK, Prelevic GM, Press M, Tuck S, O'Hare PJ, Randeva HS. Elevated concentrations of retinol-binding protein-4 (RBP-4) in gestational diabetes mellitus: Negative correlation with soluble vascular cell adhesion molecule-1 (sVCAM-1). Gynecol Endocrinol. 2008;24(6):300-5.
20. Sun L-F, Chen W-P, Li J, Ye Y-H. Correlation of retinol binding protein 4 expression in maternal serum and subcutaneous adipose tissue with insulin resistance in gestational diabetes mellitus. Zhonghua Fu Chan Ke Za Zhi. 2009:44(12):915-9.

21. Klein K, Bancher-Todesca D, Leipold H, Knofler M, Haslinger P, Handisurya A, Kautzky-Willer A, Worda C Retinol-binding protein 4 in patients with gestational diabetes mellitus. J Womens Health (Larchmt. 2010;19(3):517-21.

22. Maghbooli Z, Hossein-nezhad A, Mirzaei K, Karimi F, Besharati A, Omidfar K, Larijani B. Association between retinol-binding protein 4 concentrations and gestational diabetes mellitus and risk of developing metabolic syndrome after pregnancy. Reprod Sci. 2010;17(2):196-201.

23. Su YX, Hong J, Yan Q, Xu C, Gu WQ, Zhang YF, Shen CF, Chi ZN, Dai M,

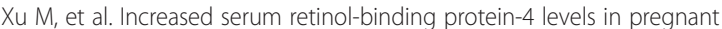
women with and without gestational diabetes mellitus. Diabetes Metab. 2010:36(6 Pt 1):470-5.

24. Tepper BJ, Kim YK, Shete V, Shabrova E, Quadro L. Serum retinol-binding protein 4 (RBP4) and retinol in a cohort of borderline obese women with and without gestational diabetes. Clin Biochem. 2010;43(3):320-3.

25. Chen Z-y, Du J. Change of serum retinol-binding protein-4 level in pregnancy obese subjects and GDM and related factors. J China Med Univ. 2011:40(5):465-8.

26. Kuzmicki M, Telejko B, Wawrusiewicz-kurylonek N, Nikolajuk A, Zwierz-gugala D, Jelski W, Kolodziejczak M, Zonenberg A, Wilczynski J, Kretowski A, et al. Retinol-binding protein 4 in adipose and placental tissue of women with gestational diabetes. Gynecol Endocrinol. 2011;27(12):1065-9.

27. Khovidhunkit W, Pruksakorn P, Plengpanich W, Tharavanij T. Retinol-binding protein 4 is not associated with insulin resistance in pregnancy. Metab Clin Exp. 2012;61(1):65-9.

28. Skvarca A, Tomazic M, Krhin B, Blagus R, Janez A. Adipocytokines and insulin resistance across various degrees of glucose tolerance in pregnancy. J Int Med Res. 2012;40(2):583-9.

29. Abetew DF, Qiu C, Fida NG, Dishi M, Hevner K, Williams MA, Enquobahrie DA. Association of retinol binding protein 4 with risk of gestational diabetes. Diabetes Res Clin Pract. 2013;99(1):48-53.

30. Nanda S, Nikoletakis G, Markova D, Poon LCY, Nicolaides KH. Maternal serum retinol-binding protein-4 at 11-13 weeks' gestation in normal and pathological pregnancies. Metabolism. 2013;62(6):814-9.

31. Liang Z, Du M, Fang Q, Chen D. Significance of RBP4 in patients with gestational diabetes mellitus: a case-control study of Han Chinese women. Gynecol Endocrinol. 2014;30(2):161-4.

32. Fruscalzo A, Londero AP, Driul L, Henze A, Tonutti L, Ceraudo M, Zanotti G, Berni R, Schweigert FJ, Raila J. First trimester concentrations of the TTRRBP4-retinol complex components as early markers of insulin-treated gestational diabetes mellitus. Clin Chem Lab Med. 2015;53(10):1643-51.

33. Du M, Wang B, Liang Z, Dong M, Chen D. The Relationship between Retinol-Binding Protein 4 Concentrations and Gestational Diabetes Mellitus in Chinese Women. Gynecol Obstet Invest. 2015. doi: 10.1159/000398794.

34. Zhang T, Zhong W (editors). Applied methodology for evidence-based medicine. Changsha, China: Central South University Press; 2011.

35. Higgins JPT, Green S (editors). Cochrane Handbook for Systematic Reviews of Interventions Version 5.1.0 [updated March 2011]. The Cochrane Collaboration, 2011. Available from http://handbook.cochrane.org/.

36. Hozo SP, Djulbegovic B, Hozo I. Estimating the mean and variance from the median, range, and the size of a sample. BMC Med Res Methodol. 2005;5:13.

37. The Expert Committee on the Diagnosis Of Diabetes Mellitus. Report of the expert committee on the diagnosis and classification of diabetes mellitus. Diabetes Care. 2003;26 Suppl 1:S5-20.

38. National Diabetes Data Group. Classification and diagnosis of diabetes mellitus and other categories of glucose intolerance. Diabetes. 1979;28(12):1039-57.

39. Department of Noncommunicable Disease Surveillance. Definition, diagnosis, and classi-fication of diabetes mellitus and its complications. Report of a WHO Consultation. Part 1: Diagnosis and classification of diabetes mellitus. WHO/NCD/NCS/99.2. Geneva: WHO; 1999.

40. Clarke GM, Anderson CA, Pettersson FH, Cardon LR, Morris AP, Zondervan KT. Basic statistical analysis in genetic case-control studies. Nat Protoc. 2011; 6(2):121-33

41. Li X, Shen L, Tan HZ. Polymorphisms and plasma level of transforming growth factor-beta 1 and risk for preeclampsia: a systematic review. Plos One. 2014;9(5):e97230.

42. Whitehead A, Whitehead J. A general parametric approach to the metaanalysis of randomized clinical trials. Stat Med. 1991;10(11):1665-77. 
43. Higgins JP, Thompson SG. Quantifying heterogeneity in a meta-analysis. Stat Med. 2002;21(11):1539-58

44. Palmer TM, Peters JL, Sutton AJ, Moreno SG. Contour-enhanced funnel plots for meta-analysis. Stata J. 2008:8(2):242-54

45. Peters JL, Sutton AJ, Jones DR, Abrams KR, Rushton L. Contour-enhanced meta-analysis funnel plots help distinguish publication bias from other causes of asymmetry. J Clin Epidemiol. 2008;61(10):991-6.

46. Meigs IB, Panhuysen Cl, Myers RH, Wilson PW, Cupples LA. A genome-wide scan for loci linked to plasma levels of glucose and $\mathrm{HbA}(1 \mathrm{c})$ in a communitybased sample of Caucasian pedigrees: The Framingham Offspring Study. Diabetes. 2002;51(3):833-40.

47. Shepherd PR, Kahn BB. Glucose transporters and insulin action-implications for insulin resistance and diabetes mellitus. N Engl J Med. 1999;341(4):248-57.

48. Hu C, Jia W, Zhang R, Wang C, Lu J, Wu H, Fang Q, Ma X, Xiang K. Effect of RBP4 gene variants on circulating RBP4 concentration and type 2 diabetes in a Chinese population. Diabet Med. 2008;25(1):11-8.

49. Hung YP, Lee NY, Lin SH, Chang HC, Wu CJ, Chang CM, Chen PL, Lin HJ, Wu YH, Tsai PJ, et al. Effects of PPARgamma and RBP4 gene variants on metabolic syndrome in HIV-infected patients with anti-retroviral therapy. PLoS One. 2012;7(11):e49102.

50. Liu J, Gao JY, Zhang JP, Li PQ, Liu JX, Liu J, Xie XD. Evaluation of the association between retinal binding protein 4 polymorphisms and type 2 diabetes in Chinese by DHPLC. Endocrine. 2008;34(1-3):23-8.

51. Wu Y, Li H, Loos RJ, Qi Q, Hu FB, Liu Y, Lin X. RBP4 variants are significantly associated with plasma RBP4 levels and hypertriglyceridemia risk in Chinese Hans. J Lipid Res. 2009;50(7):1479-86.

52. Nair AK, Sugunan D, Kumar H, Anilkumar G. Case-control analysis of SNPs in GLUT4, RBP4 and STRA6: association of SNPs in STRA6 with type 2 diabetes in a South Indian population. PLoS One. 2010;5(7):e11444.

53. Munkhtulga L, Nakayama K, Utsumi N, Yanagisawa Y, Gotoh T, Omi T, Kumada M, Erdenebulgan B, Zolzaya K, Lkhagvasuren T, et al. Identification of a regulatory SNP in the retinol binding protein 4 gene associated with type 2 diabetes in Mongolia. Hum Genet. 2007;120(6):879-88.

54. Craig RL, Chu WS, Elbein SC. Retinol binding protein 4 as a candidate gene for type 2 diabetes and prediabetic intermediate traits. Mol Genet Metab. 2007;90(3):338-44.

55. Kovacs P, Geyer M, Berndt J, Kloting N, Graham TE, Bottcher Y, Enigk B, Tonjes A, Schleinitz D, Schon MR, et al. Effects of genetic variation in the human retinol binding protein-4 gene (RBP4) on insulin resistance and fat depot-specific mRNA expression. Diabetes. 2007;56(12):3095-100.

56. van Hoek M, Dehghan A, Zillikens MC, Hofman A, Witteman JC, Sijbrands EJ. An RBP4 promoter polymorphism increases risk of type 2 diabetes. Diabetologia. 2008:51(8):1423-8.

57. Munkhtulga L, Nagashima S, Nakayama K, Utsumi N, Yanagisawa Y, Gotoh T, Omi T, Kumada M, Zolzaya K, Lkhagvasuren T, et al. Regulatory SNP in the RBP4 gene modified the expression in adipocytes and associated with BMI. Obesity (Silver Spring). 2010;18(5):1006-14.

\section{Submit your next manuscript to BioMed Central and we will help you at every step:}

- We accept pre-submission inquiries

- Our selector tool helps you to find the most relevant journal

- We provide round the clock customer support

- Convenient online submission

- Thorough peer review

- Inclusion in PubMed and all major indexing services

- Maximum visibility for your research

Submit your manuscript at www.biomedcentral.com/submit 